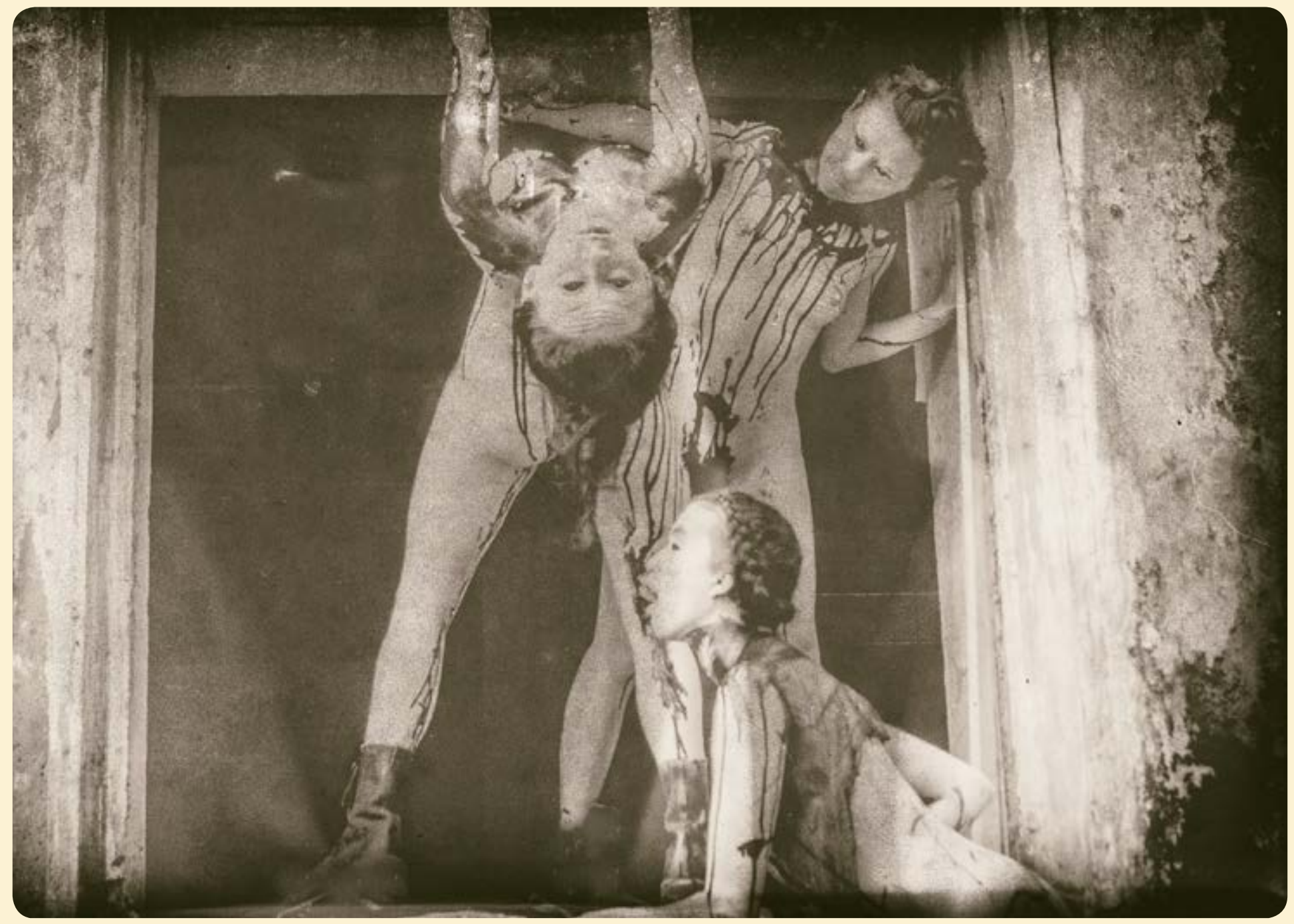

C.A.R.N.Experimento. Meta Carne. Performance (2015). PH. Jennyfer Caro. Edición: Raúl Vidales. 


\section{Cuerpos asimétricos en movimiento'}

\section{Asymmetric bodies in motion // Corpos assimétricos em movimento}

Ángela María Hoyos Quintero²

Universidad Santiago de Cali, Colombia

angela.hoyos@correounivalle.edu.co

Revista Corpo-grafías: Estudios críticos de y desde los cuerpos / Volumen 7 - Número 7 / Enero - diciembre 2020 / ISSN Impreso 2390-0288, ISSN Digital 2590-9398 / Bogotá, D.C., Colombia / 17-28

Fecha de recepción: 15 de enero de 2019

Fecha de aceptación: 1 de mayo de 2019

DOI: https://doi.org/10.14483/25909398.15502

Cómo citar este artículo: Hoyos, A. (2020, enero-diciembre). Cuerpos asimétricos en movimiento. Revista Corpo-grafías: Estudios críticos de y desde los cuerpos, 7(7), pp. 17-28 / ISSN 2390-0288.

\footnotetext{
$1 \quad$ Artículo de investigación: El presente artículo deriva de la investigación realizada por la autora, dentro de un proyecto de investigación liderado por el profesor Armando Collazos de la Universidad del Valle, cuyo resultado principal fue el montaje de las obras de teatro físico "Como se salvó Wang Fo" y "El vuelo de la Alondra" en los años 2015 y 2017, en dicho proceso fue relevante la participación de diferentes profesionales que aportaron sus conocimientos para una concepción holística del actor.

2 Magister en Epidemiología y Fisioterapeuta, Universidad del Valle. Docente, Programa fisioterapia, Universidad Santiago de Cali. Docente kinesiología de la danza, Licenciatura en Danza Clásica, Universidad del Valle. Miembro grupo investigación INCIDE, Universidad del Valle. Ha tenido un constante interés por el estudio del movimiento, combinando su experiencia como fisioterapeuta con la práctica de Aikido.
} 


\section{Resumen}

La participación del área de la salud, en los proyectos de investigación del colectivo Voces del Cuerpo, se orienta tras una necesidad latente. Objetivo: indagar nuevas formas de concebir y abordar al artista corporal desde el punto de vista de la relación cuerpo-salud redundando positivamente en el resultado artístico. Metodología: el proceso se realizó en 5 etapas: observación, valoraciones individuales, aplicación de cuestionarios, discusiones grupales y análisis de los resultados. Resultados: más del $80 \%$ de los performer presentaban asimetría corporal inicial, observando pocas diferencias post-intervención (33.33\%), esto aumenta el riesgo de presentar lesiones y deformidades, pero es potencializado por los performer en la construcción del personaje. Respecto a la calidad de vida, el factor que genera mayor efecto es la inestabilidad económica. Conclusiones: El análisis de la simetría corporal permitió construir una base para la orientación del trabajo corporal del colectivo y la mejora de la conciencia corporal.

\section{Palabras clave}

Postura; fuerza muscular; elasticidad muscular; propiocepción; cuidado corporal; calidad de vida

\section{Abstract}

The participation of the health area, in the research projects of the Voces del Cuerpo collective, is guided by the latent need. Objective: to explore new ways of conceiving and approaching the body artist from the point of view of the body-health relationship, resulting positively in the artistic result. Methodology: the process was carried out in 5 stages: observation, individual assessments, application of questionnaires, group discussions and analysis of the results. Results: more than $80 \%$ of the performers presented initial body asymmetry, observing few post-intervention differences (33.33\%), this increases the risk of presenting injuries and deformities, but is enhanced by the performers in the construction of the character. Regarding the quality of life, the factor that generates the greatest effect is economic instability. Conclusions: The analysis of body symmetry allowed to build a basis for the orientation of the collective body work and the improvement of body awareness.

\section{Keywords}

Posture; muscular strength; muscular elasticity; proprioception; body care; quality of life

\section{Resumo}

A participação da área da saúde, nos projetos de pesquisa do coletivo Voces del Cuerpo, é pautada pela necessidade latente. Objetivo: explorar novas formas de conceber e abordar o artista corporal a partir do ponto de vista da relação corpo-saúde, resultando positivamente no resultado artístico. Metodologia: o processo foi realizado em 5 etapas: observação, avaliações individuais, aplicação de questionários, discussões em grupo e análise dos resultados. Resultados: mais de $80 \%$ dos artistas apresentaram assimetria corporal inicial, observando poucas diferenças pós-intervenção $(33,33 \%)$, isso aumenta o risco de apresentar lesões e deformidades, mas é reforçada pelos performers na construção do personagem. Em relação à qualidade de vida, o fator que gera maior efeito é a instabilidade econômica. Conclusões: A análise da simetria corporal permitiu construir uma base para a orientação do trabalho corporal coletivo e a melhoria da consciência corporal. 


\section{Palavras-chaves}

Postura; força muscular; elasticidade muscular; propriocepção; cuidados com o corpo; qualidade de vida

La participación en los proyectos de investigación del colectivo Voces del Cuerpo -pesquisa para la creación corporal escénica -, permitió indagar desde el área de la salud acerca de la condición corporal de los performers, su dificultad de acercarse a los conceptos de salud y cómo mejorar su conciencia corporal.

Durante la investigación, se planteó que los conocimientos sobre la composición corporal le permitirían al performer mejorar la conciencia de su cuerpo, donde "el estudio de la biomecánica aplicada ... provocaría mejoras en el entrenamiento y en las técnicas del movimiento" (Shippen, 2012, pp. 39-43) y sumado a un entrenamiento riguroso de la fuerza, la elasticidad muscular y la propiocepción permitiría a los actores, artistas del cuerpo y performers tener conocimiento de sus limitaciones y posibilidades y así adquirir la conciencia de un cuerpo sano, lo que resulta fundamental para el teatro físico. Mientras existe tensión no se puede hablar de sensaciones sutiles, correctas, ni de una vida espiritual normal del personaje. Por eso antes de iniciar la creación, hay que poner en orden los músculos para que no paralicen la libertad de acción (Stanislavski, 2003).

Ésta investigación se llevó a cabo durante los procesos de montaje de "Cómo se salvó Wang Fo", una adaptación del cuento de Marguerite Yourcenar y "El vuelo de la alondra", una adaptación de Casa de muñecas de Henrik Ibsen. La intervención ocurrió en el momento en que el director determinó las unidades de acción de cada una de las escenas, tiempo durante el cual los actores ya habían empezado diferentes exploraciones de las técnicas de movimiento usadas para el montaje. Durante este proceso, se realizaron observaciones del colectivo en sus sesiones de training corporal, evaluaciones que permitieron realizar aportes significativos desde la valoración postural de cada uno de los integrantes correlacionando la presencia de asimetría corporal con el riesgo de lesión y las particularidades de cada uno de los actores en el momento de exponerse a las diferentes técnicas de movimiento estudiadas $y$, finalmente, se plantearon recomendaciones respecto al fortalecimiento y al trabajo propioceptivo con el ánimo de mejorar la conciencia corporal, que a pesar de ser en algunos momentos un concepto gaseoso engloba la intervención realizada, ya que “... El objetivo del cuerpo que se organiza para moverse con el mínimo esfuerzo y la máxima eficiencia, no es la fuerza muscular sino el aumento de la conciencia al hacerlo" (Feldenkrais, 1995, p. 209). Adicional a esto se indagó respecto a la calidad de vida de los performer y cómo diferentes factores pueden influir en su desempeño pues “...además de movimiento del cuerpo, la danza es la construcción de un cuerpo de acciones, donde el cuerpo se entiende en términos del resultado de su inclusión en el orden significante" (Escudero, 2012, p. 108). Así, cualquier movimiento es la suma de diferentes posturas que al unirse de manera armónica permiten crearlo y por ende, si la postura se encuentra alterada, sea cual sea la causa, el movimiento no resultará fluido y armónico.

Para realizar el proceso investigativo se planteó como objetivo indagar nuevas formas de concebir y abordar al artista corporal desde el punto de vista de la relación 


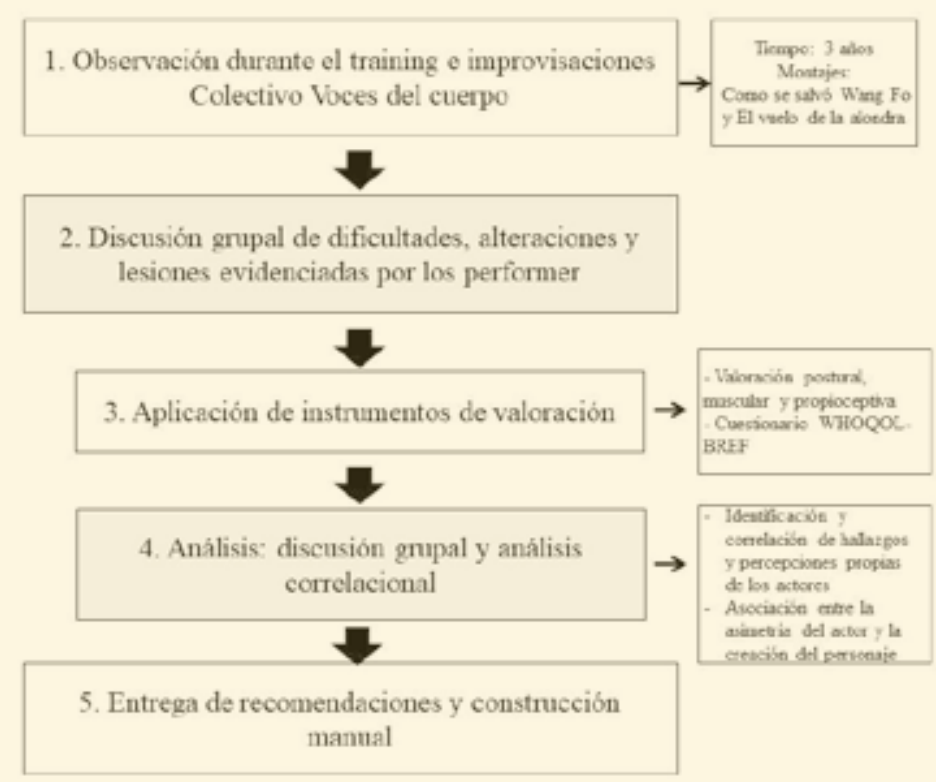

Figura 1. Metodología del proceso investigativo.

cuerpo-salud, con la clara pretensión de redundar positivamente en el resultado artístico final, para ello se plantearon diferentes objetivos metodológicos donde se buscó caracterizar el estado postural, muscular y propioceptivo inicial de los performers a través de valoraciones posturales y de pruebas de fuerza muscular e identificar el nivel de calidad de vida de los integrantes del colectivo, para así analizar los hallazgos relacionándolos con su desempeño y finalmente entregar las recomendaciones que se compilaron en un manual de cuidado corporal para ser usado por ellos.

Este encuentro permitió reflexionar sobre el movimiento humano como un continuo ajuste del cuerpo en contra de la gravedad para mantener el equilibrio, requiriendo acomodaciones constantes del sistema artro-muscular para erguirse sin dificultad y controlar los movimientos al pasar de un plano a otro, reconociendo las diferentes dimensiones del movimiento para adquirir un equilibrio relativamente estable o un desequilibrio permanente, constantemente compensado, como argumenta Hernández (1995) y como un proceso influenciado por factores externos, como los factores psicológicos, emocionales y sociales, facilitando en los performers el reconocimiento de limitaciones o alteraciones que generaban dificultades durante la exploración del movimiento. 


\section{Metodología}

La investigación se llevó a cabo durante los procesos de montaje referidos planteándose cinco etapas para la indagación desde el área de la salud:

En la primera etapa, se realizó observación directa del movimiento y de los ajustes realizados por los performers durante su entrenamiento y la etapa de improvisación, ésta información fue registrada en un formato preestablecido lo que permitió correlacionarlo posteriormente con los resultados de la valoración individual.

En la segunda etapa, la discusión con los integrantes del colectivo brindó una visión global de la conceptualización del cuerpo que tenían los performers y sus conocimientos previos. Sumado a esto se tuvo en cuenta el tiempo y tipo de práctica corporal que cada uno había realizado. (Véase figura 1) En la tercera etapa, la valoración individual permitió como fisioterapeuta-epidemióloga, completar el análisis postural, muscular y propioceptivo y analizar la calidad de vida con la aplicación del cuestionario WHOQOL-BREF, identificando en cada uno de los integrantes del colectivo, particularidades que se relacionarían con su estado corporal.

En la cuarta etapa, se realizó una discusión con el colectivo sobre los hallazgos, lo que permitió construir su estado de base respecto a la condición corporal y la calidad de vida, identificando las principales dificultades y fortalezas y correlacionando estos hallazgos con su desempeño como performers en los montajes en cuestión. Esta etapa se extendió hasta después del estreno de las obras, donde a través de registros fílmicos y fotográficos se realizó el análisis de la corporalidad del personaje buscando la existencia de asociaciones entre la asimetría corporal del actor y la creación física de su personaje, valorando la habilidad de explorar las dificultades o alteraciones y usarlas a su favor en este proceso.

Finalmente, en la quinta etapa, se realizó entrega de las recomendaciones de fortalecimiento, estiramientos, trabajo propioceptivo y pliométrico generadas por el análisis previo de la condición corporal, información que se compiló en un manual de cuidado corporal. Este diseño metodológico permitió hacer un análisis de diferentes aspectos de los performers generando recomendaciones pertinentes para que a partir de sus fortalezas y debilidades lograran construir un lenguaje corporal, estético y armónico en conjunto.

\section{Resultados}

El principal resultado de todo el proceso fue el estreno de las obras, (entre 2015 y 2017) "Cómo se salvó Wang Fo" y "El vuelo de la Alondra", donde confluimos diversos profesionales. Respecto al área de la salud, el resultado principal fue el análisis de la condición corporal de los integrantes del colectivo desde el punto de vista de la relación cuerpo-salud.

Como resultados secundarios, se encuentra la caracterización del colectivo respecto a la alineación postural (tabla 1), el análisis del efecto de la asimetría corporal de cada uno de los actores para la construcción del personaje y el análisis de la calidad de vida de los integrantes del colectivo.

\section{Alineación postural, simetría corporal y efectos}

En este sentido, más de la mitad de los evaluados presentaron alteración en su centro de gravedad, lo cual genera una distribución inadecuada del peso, en su mayoría de manera anterior, produciendo sobrecarga 
en las rodillas y los hallux, articulaciones donde se hace evidente dichas alteraciones. La idea es prevenir futuros problemas ya que, en caso de persistir esta alteración, el cuerpo tendrá que realizar acomodaciones a nivel toracolumbar y pélvico para posicionarse en contra de la gravedad. Los efectos pueden no observarse tempranamente pero un cuerpo que persiste en esta postura inadecuada genera, en el futuro, problemas articulares permanentes, situación que aumenta el riesgo de esguinces, luxaciones y tendinitis. De la misma manera, la asimetría de la cintura escapular y del cuello provoca alteraciones en la distribución del peso corporal, pues la percepción del propio cuerpo tiene directa relación con la orientación de la mirada. Es así como, el lado hacia donde el ser humano oriente la mirada y rote su cabeza, es el lado que más reconoce y el que recibe más peso. Si se analiza el uso de los músculos como herramienta para mantenerse en contra de la gravedad, el hemicuerpo que carga el peso será el de mayor fortalecimiento y propiocepción, lo que induce el riesgo de lesión en el lado contralateral.

Aunque la intervención se orientó usando el entrenamiento autodirigido que delega a los performer la responsabilidad de la aplicación de las recomendaciones individuales (sugeridas en el manual), fue inevitable que apareciesen dificultades asociadas a este modelo de entrenamiento, como por ejemplo componentes emocionales los cuales retrasan su realización. Aun así, se observaron pequeñas mejorías en la simetría de los performers, en relación con la distribución del peso y su autopercepción, obteniendo que ellos identificaran sus alteraciones y lograran pequeñas acomodaciones temporales. Es pertinente aclarar que esta evidencia requiere un mayor tiempo de intervención ya que dicha habilidad se adquiere a través de un trabajo constante, perseverante y disciplinado, que en la mayoría de los casos se afianza después de muchos años, constituyéndose prácticamente en una indagación permanente.

\begin{tabular}{|c|c|c|c|c|}
\hline \multirow{2}{*}{ Aspectos valoración postural } & \multicolumn{2}{|c|}{ Valoración inicial (n=6) } & \multicolumn{2}{|c|}{ Valoración final $(n=6)$} \\
\hline & $\mathrm{Si}$ & No & Si & No \\
\hline Asimetría cabeza & $50 \%(3)$ & $50 \%(3)$ & $33.33 \%(2)$ & $66.66 \%(4)$ \\
\hline $\begin{array}{l}\text { Asimetría superior (cintura esca- } \\
\text { pular y hombros) }\end{array}$ & $83.33 \%(5)$ & $16.66 \%(1)$ & $83.33 \%(5)$ & $16.66 \%(1)$ \\
\hline $\begin{array}{l}\text { Asimetría inferior (cintura pélvica } \\
\text { y miembros inferiores) }\end{array}$ & $66.66 \%(4)$ & $33.33 \%(2)$ & $66.66 \%(4)$ & $33.33 \%(2)$ \\
\hline Alteración columna & $83.33 \%(5)$ & $16.66 \%(1)$ & $50 \%(3)$ & $50 \%(3)$ \\
\hline
\end{tabular}

Tabla 1. Resultados de valoración postural detectados durante la investigación. 


\section{Caso 1. Personaje Wang Fo}

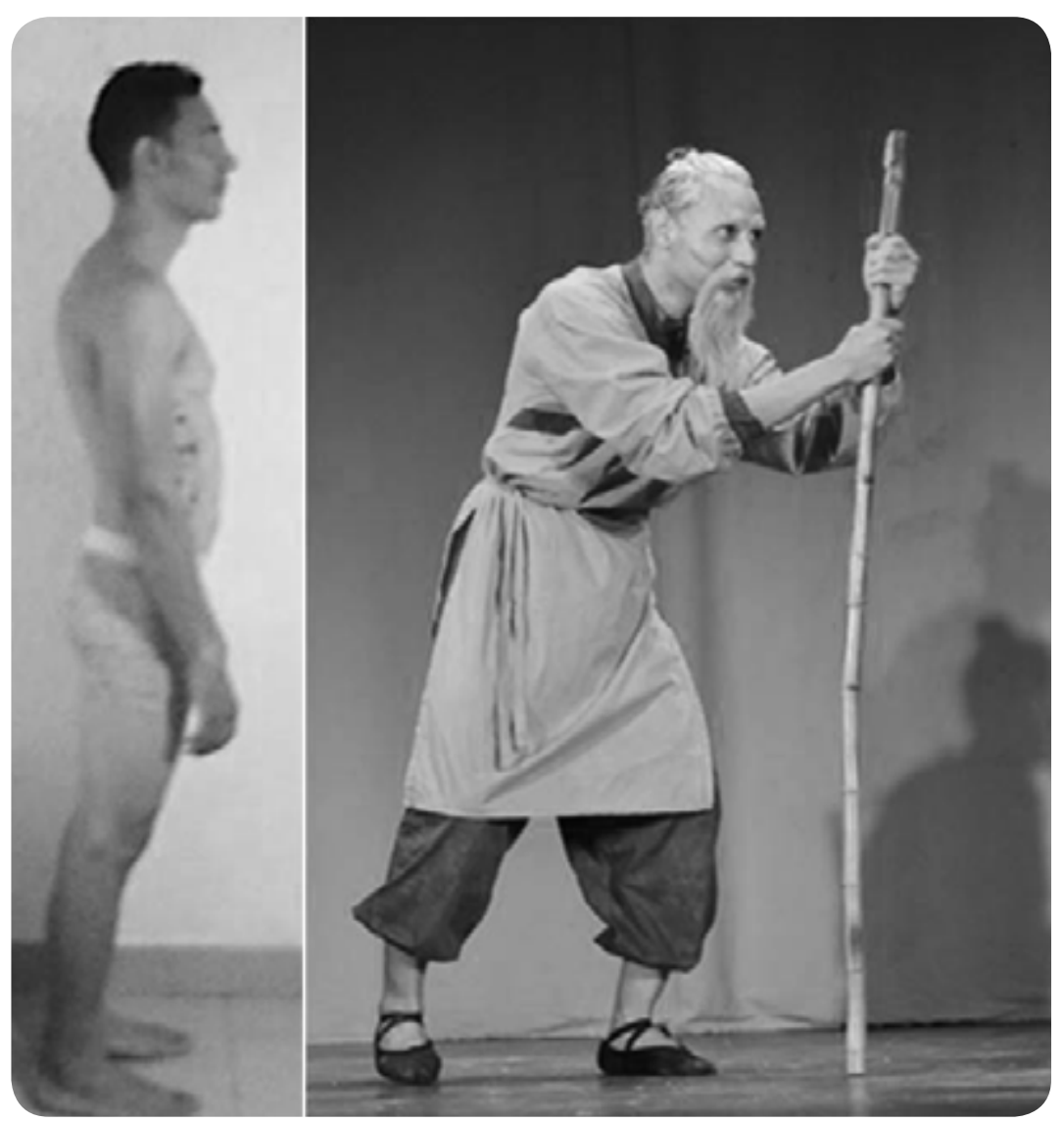

Imagen 1. Actor y Personaje. Cómo se salvó Wang Fo (obra de teatro). Archivo de la autora del artículo.

\section{Efecto de la asimetría corporal en la construcción del personaje}

(Véase tabla 1) El análisis de la alineación postural de los actores y la asociación de estas alteraciones con el efecto directo en sus tejidos blandos y por ende en la estructura, permitió en esta investigación encontrar puntos de conexión entre el actor y el personaje interpretado. Aunque la asimetría genera un efecto negativo en el cuerpo de los actores, la capacidad de utilizar o potenciar las alteraciones para la construcción del personaje posibilitó a los actores disminuir los riesgos de lesión. Para el análisis en mención se tomaron dos casos donde la asimetría corporal es evidente mada a otras alteraciones posturales que generan mayor riesgo para el actor. 


\section{Caso 2. Personaje Doctor Rank}

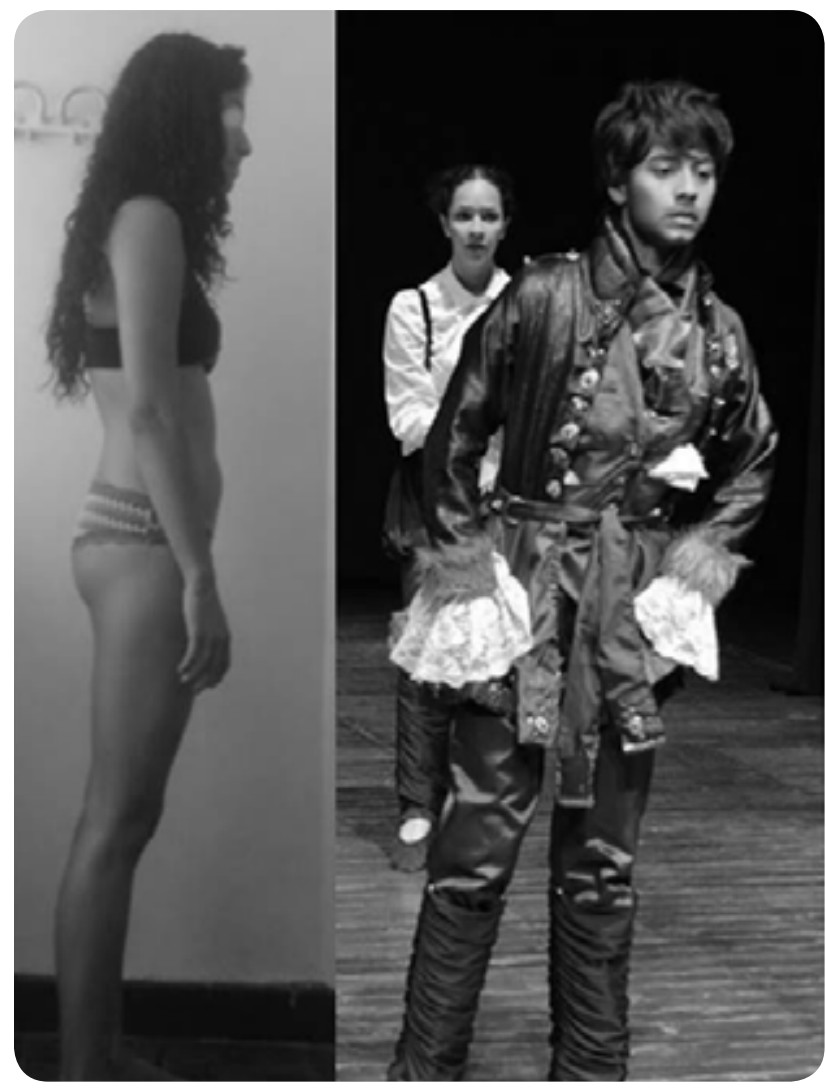

Imagen 2. Actriz y personaje. El vuelo de la Alondra (obra de teatro). Archivo de la autora del artículo

En el caso del personaje Wang Fo, de la obra de Yourcenar, se observa en el actor un aumento de la cifosis torácica, centro de gravedad y cabeza adelantados, generando tensión muscular en los músculos del cuello, pectoral y tibial anterior. Estas tensiones sumadas a la dificultad que presentaba este actor, en particular, de relajar adecuadamente sus músculos tanto en reposo como en actividad, evidenciando restricción en las fascias toracolumbares y llevaron a que la construcción de su personaje, quien es un anciano pintor, lo representara haciendo uso de una postura flejada pero con alta tonicidad, justificando la realización de movimientos rápidos y agiles, sin perder la postura característica del personaje. Fue así como las alteraciones posturales de base, las tensiones musculares y restricciones, le permitieron construir un personaje que, a los ojos del público, se observa como lo propuso el actor. En el caso del personaje Wang Fo, de la obra de Yourcenar, se 
observa en el actor un aumento de la cifosis torácica, centro de gravedad y cabeza adelantados, generando tensión muscular en los músculos del cuello, pectoral y tibial anterior.

El segundo caso, es el personaje Doctor Rank, de la obra "El vuelo de la Alondra", donde la postura de base de la actriz se evidencia como una postura inactiva, tronco flejado, hipotonico, hombros rotados internamente y adelantados, asi como el centro de gravedad. Cabe anotar que siendo el doctor Rank un personaje masculino, de manera antagonica, la naturaleza femenina de la actriz aporta a la concepción que ella propone para el personaje. Este personaje se encuentra enfermo y al borde de la muerte, en este caso tambien, la actriz logra potenciar sus alteraciones en favor del personaje, quien es un hombre con poca energia, caminar lento y tronco poco activo por la tuberculosis que le aqueja. El uso de la hipoactividad muscular de base para la interpretación del personaje permite que el público comprenda desde el primer momento que el Doctor Rank es un

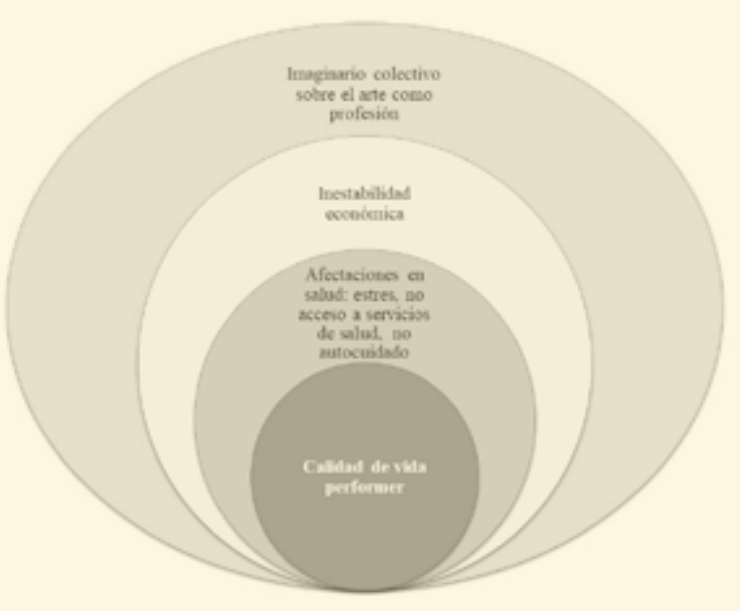

Figura 2. Análisis de la asociación entre la calidad de vida y la salud de los performers en la investigación.

hombre enfermo, con dolor y dificultad para respirar adecuadamente (según comentarios del público).

\section{Análisis de la calidad de vida}

Respecto a la calidad de vida se observó que, para la mayoría, esta es afectada por la dificultad de controlar factores económicos que están relacionados directamente con su vida laboral, evidenciando la dificultad de ejercer el arte como medio productivo en este contexto social. Los efectos negativos de este tipo de inconformidades en el bienestar de las personas han sido abordados en diferentes estudios vigentes (Wilkinson, 2003) relacionando el aumento de los niveles de estrés con el riesgo de enfermedad. Lo anterior se aplicó y se relacionó directamente con las lesiones 
y alteraciones que presentaron los performers del colectivo durante el desarrollo del proyecto, pues al no tener una adecuada percepción de ésta y una cultura de autocuidado, asumieron riesgos que incidieron de forma negativa en su salud. Para el análisis se tuvo en cuenta el efecto de factores externos en la calidad de vida de los performers (figura 2), donde se puede suponer que el imaginario colectivo sobre el arte como profesión, afecta las oportunidades que tienen los performers de tener estabilidad económica, convirtiéndose en un detonante de muchos otros aspectos que influyen en los procesos de autocuidado y salud. A pesar de que, en el proceso se implementaron correctivos paliativos que posibilitaron el desarrollo y la terminación del proyecto, pues los actores que mejoraron su estabilidad corporal producto del incremento en la fuerza muscular y la propiocepción evidenciaron mayor seguridad y libertad y que a través de los cambios que hicieron evidente cada uno de los actores se observaron igualmente transformaciones en la corporalidad del personaje, este es un proceso que requiere la intervención de otras áreas del conocimiento para la generación de un efecto a largo plazo en el desempeño de los artistas.

(Véase Figura 2) Las consideraciones precedentes en el contexto de la investigación, generaron inquietudes sobre el abordaje de los colectivos escénicos y sobre la importancia de que este se realice por parte de un equipo profesional interdisciplinario que trabaje mancomunadamente, buscando desde un saber más amplio, disminuir el riesgo en el que se encuentran.

Dado que la asimetría ocupó un lugar importante en esta investigación, vale la pena preguntarse si la alteración en la calidad de vida podría presentarse como una asimetría también,más a nivel emocional, que genera igualmente efectos negativos en el actor y que redunda negativamente en su ejercicio profesional y su rol como sujeto social en el entorno.

Como resultado del análisis postural y los efectos que las alteraciones generan en la estructura, se presentó un manual (en proceso de publicación) que contiene orientaciones sobre fortalecimiento muscular, correcciones posturales y entrenamiento propioceptivo, material educativo del cual pueden hacer uso estudiantes, artistas o profesionales de la escena que estén en el ejercicio y aplicación de expresiones escénicas corporales y físicas como apoyo a su trabajo de creación.

\section{Discusión}

Aunque la intervención desde el área de la salud en las actividades del colectivo escénico Voces del cuerpo, de la ciudad de Cali, Colombia, permitió sensibilizar a los integrantes y a los demás profesionales que participaron en los procesos de montaje, no se diseñó una estrategia para compartir los resultados y sugerencias a otros colectivos escénicos de la ciudad. Es posible que estos resultados no sean generalizables para el mundo, dadas las características del país en el que se realizó el estudio.

Para Pérez (2011), la existencia de ciertos patrones - posturales, motrices y expresivos - en los actores, determinan en cierta medida el tipo de expresividad del sujeto y forman parte de su identidad pero pueden estructurarse de tal forma que limitan el desarrollo expresivo y perturban el proceso de creación del actor. En esta investigación, se evidencia la afectación desde el área subjetiva y la habilidad que ha adquirido el actor de potenciar su creación a partir de sus deficiencias.

Sin embargo, lo más relevante en esta investigación es la importancia que tienen esos aspectos intangibles 
desde el área emocional y socio-económica que favorecen la independencia y el entusiasmo al realizar un entrenamiento corporal, y la adquisición de hábitos saludables, lo que requiere tiempo y dedicación.

\section{Conclusiones}

Respecto a la relación cuerpo-salud, la identificación y el análisis de la simetría corporal permitió construir una base para la orientación del trabajo corporal del colectivo, relacionado directamente con el balance corporal y su efecto en la alineación de un cuerpo en el espacio, siendo significativo para el performer tanto en la interpretación como en su salud, esto vinculado a una visión holística del ser humano

La intervención del área de la salud en el proceso de montaje brindó herramientas precisas respecto al análisis corporal de los performers que le permitieron mejorar la fuerza y elasticidad muscular y la propiocepción disminuyendo el riesgo de lesión y aumentando la conciencia corporal, esta conciencia que se replica a nivel postural y emocional genera en el actor la posibilidad de mejorar en expresividad, en libertad de movimiento, en precisión técnica y por ende aumentar considerablemente la seguridad durante los movimientos de riesgo El trabajo en la conciencia debe ser sistemático e ininterrumpido requiriendo un compromiso personal del performer para una práctica constante y sincera que le permita mantener los logros alcanzados y continuar su proceso.

La construcción de un personaje es un proceso dinámico y puede contener características del actor que lo personifica, si logra identificar en su estructura aspectos que van en concordancia con el personaje o que pueden aportar a la imagen física del mismo, el actor debe lograr transformarse en tal medida que su presencia en el escenario se convierta en una sola, logrando no ser un actor representando sino el personaje mismo. Esto solo se logra en el momento que ligado a su entrenamiento corporal, el performer vincule el trabajo de conciencia postural y corporal, que como ya se ha dicho debe ser permanente

El estudio desde el área de la salud de los colectivos escénicos requiere de la participación de un equipo profesional interdisciplinario que permita generar una visión más amplia de su problemática. Y facilite la construcción de procesos que propendan a la mejoría del estado de salud de los integrantes y a su calidad de vida que como se mencionó en el documento, depende de múltiples factores, entre ellos los factores económicos, el posicionamiento a nivel social como profesionales, la autoconcepción de ser seres vulnerables que requieren una orientación especifica respecto a las conductas de riesgo corporal y quienes deben transformar la visión de sí mismos para lograr adquirir conductas de autocuidado.

Este proyecto generó la construcción de una línea de investigación que permite integrar el arte y la ciencia hacia un fin común.

\section{Referencias}

Escudero, M. C. (2012). Consideraciones epistemológicoconceptuales para el estudio del cuerpo en la Danza. Revista Latinoamericana de Metodología de Ciencias Sociales. 2(1), La Plata.

Hernández, J. (1995). Torpeza motriz. Barcelona: EUB.

Shippen, J. (2012). A Kinematic Approach to Calculating Ground Reaction Forces in Dance. Journal of Dance Medicine \& Science. 16(1), pp.39-43, Reino Unido. 
Stanislavski, C. (2003). La preparación del actor. Madrid: La avispa.

Wilkinson, R. G., Marmot, M. (Eds.). (2003). Social determinants of health: the solid facts. World Health Organization. Copenhagen, Denmark: The regional office for Europe of the WHO.

Pérez Cubas, G. (2011). Cuerpos con sombra. (Pról.). Disponible en: http://www.terapiadelmovimientoavanzada.es/tratamientoy-reeducacion-postural-y-del-movimiento-en-artes-escenicas/. Consultado: 15-01-2019. 

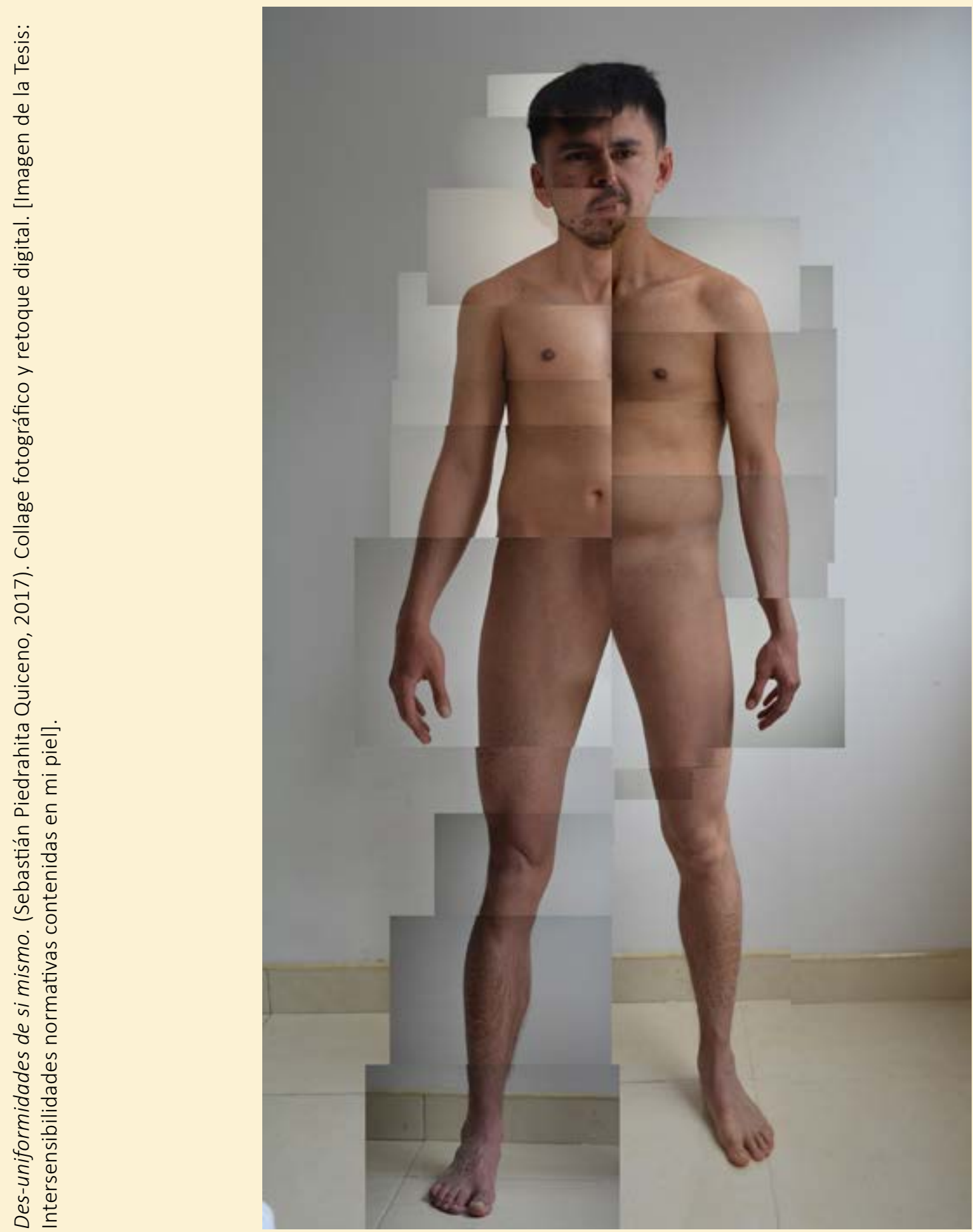